\title{
Une ekphrasis paradoxale des statues du Belvédère dans les 'Vingt-quatre sonnets romains' de Jacques Grévin
}

\section{Michèle Clément}

\section{(2) OpenEdition}

Journals

\section{Édition électronique}

URL : http://journals.openedition.org/studifrancesi/35558

DOI : 10.4000/studifrancesi.35558

ISSN : 2421-5856

Éditeur

Rosenberg \& Sellier

\section{Édition imprimée}

Date de publication : 1 juillet 2005

Pagination : 49-60

ISSN : 0039-2944

\section{Référence électronique}

Michèle Clément, "Une ekphrasis paradoxale des statues du Belvédère dans les 'Vingt-quatre sonnets romains' de Jacques Grévin », Studi Francesi [En ligne], 145 (XLIX | I) | 2005, mis en ligne le 30 novembre 2015, consulté le 20 avril 2021. URL : http://journals.openedition.org/studifrancesi/35558 ; DOI : https://doi.org/10.4000/studifrancesi.35558

\section{(c)}

Studi Francesi è distribuita con Licenza Creative Commons Attribuzione - Non commerciale - Non opere derivate 4.0 Internazionale. 


\section{Une ekphrasis paradoxaledes statues du Belvédère dans les 'Vingt-quatre sonnets romains' de Jacques Grévin}

Jacques Grévin, docteur en médecine, régent de la Faculté de médecine de Paris, auteur de tragédie et de comédies remarquées par Ronsard, auteur de deux recueils poétiques, L'Olympe et $L a$ Gélodacrye, vantés par les poètes de la Pléiade, avait entamé une carrière de poète prometteuse en France entre 1558 et 1561.

Son passage à la Réforme, rendu public aux alentours de 1560-61, entraîna de nombreuses conséquences dans sa vie qui eurent des répercussions sur son œuvre : désavoué par Ronsard qui raya son nom de ses œuvres, rayé des registres de la Faculté de Médecine de Paris, obligé de quitter le royaume de France à plusieurs reprises, il voyage et séjourne à Londres auprès de la reine Elisabeth, puis à Anvers chez l'imprimeur Christophe Plantin, de nouveau à Londres et obtient finalement en 1568 un poste de médecin à Turin auprès de Marguerite de Savoie, poste qu'il conservera jusqu'à sa mort prématurée à 32 ans, en novembre 1570. C'est pendant ces presque trois années passées au service de Marguerite de Savoie qu'il fit un voyage à Rome ${ }^{1}$. Ce voyage donna lieu à la rédaction des vingt-quatre sonnets romains, dédiés à Marguerite de Savoie, et restés manuscrits jusqu'à la fin du XIXe siècle².

Ces vingt-quatre sonnets romains sont largement redevables aux Antiquités de Du Bellay, recueil publié en 1558. A la façon des Antiquités, le recueil se présente d'abord comme une topographie de la Ville où le constat des ruines architecturales entraîne une méditation sur le temps et la dégradation des empires. Au sein du massif des 24 sonnets, six se détachent parce qu'ils ne renvoient pas aux monuments architecturaux de Rome tombés en ruine mais aux statues du Belvédère: la topographie se fait ekphrasis.

Que se passe-t-il dans le texte quand le poète change de méthode de représentation du réel? de la topographie à l'ekphrasis, la concentration du regard et l'animation des statues modifient-t-elles le jugement porté sur l'objet romain?

Ces six sonnets font allusion à huit des statues du Cortile delle statue dans le jardin du Belvédère au Vatican, et plus précisément font allusion au giardino segreto

(1) Peut-être fin 1569, à l'occasion de démélés entre le pape Pie V et Emmanuel-Philibert, le duc de Savoie, qui voyait d'un mauvais œil Cosme de Medicis élévé au rang de grand duc de Toscane par le pape; une ambassade est bien attestée fin 69 avec réponse du pape en janvier 70 selon Samuel Guichenon, dans Histoire généalogique de la Maison de Savoie, 1660, T. II, p. 689 (fac similé, éd. Horvath,
Roanne). Rien ne prouve que Grévin en était.

(2) On les trouve édités par L. PINVERT dans sa monographie, Jacques Grévin, (1538-1570), étude biographique et littéraire, Paris, Fontemoing, 1899, pp. 358-370. Je les ai édités plus récemment avec $L a$ Gélodacrye, Publications de l'Université de SaintEtienne, 2000, pp. 117-134. 
17 Vous qui venez à Rome, et qui soigneusement La cause recherchez de sa grande ruine, Voyez des deux Venus la sculpture divine Au jardin où le pape entre secrettement.

Voyez là Cleopatre, et le vieil bastiment Du temple de Venus, de Flore et de Faustine ; Sçachez qu'Enée estoit fils d'une concubine, Ainsi que Romulus qui fit son fondement.

Une seule Venus, une Heleine ravie, Firent perdre aux Troyens l'Estat avec la vie, Et firent ruiner ce que Priam avoit.

Ne vous estonnez doncq si ces rues sont pleines De palais ruinez, puisque dans Rome on voit Presqu'une infinité de Venus et d'Heleines.

18 Tu te doibs contenter, ô grand'Roine d'Egypte, Cleopatre excellente en richesse et beauté, Tu te dois contenter de voir ceste Cité Du chef au fondement piteusement destruite.

Tu la vois aujourd'huy et chetive et reduite En un malheur si grand, que sa principauté Se couvre du manteau de froide pauvreté Qui a quitté le Ciel, et la Terre seduitte.

Or contente toy doncq, bien qu'Auguste jadis T'aye arraché des mains le sceptre que perdis Pour avoir trop aimé le valeureux Antoine ;

Car le temps, plus puissant que ne sont les humains

A fin de te vanger te faict voir les Romains Reduicts piteusement sous le pouvoir d'un moine.

19 Le grand Laocoon, prophete infortuné, Cognoissant des Gregeoiz la nature traistresse, Frappa, tenant au poing la lance vangeresse, Le cheval qui portoit le malheur soubçonné.

Pallas, ou le destin contre luy mutiné, Fit miserablement devorer sa vieillesse Par deux serpens tortus, et soudain par la Grece Troye fut mise en cendre et Priam ruiné.

La mort de ce vieillard incontinent suivie Par le sac des Troyens, et de toute l'Asie, Monstre assez combien peut ce signe ruineux.

Les Romains ruinez se ruinent encore, Car ils ont retenu tousjours au milieu d'eulx Un vieil Laocoon que le serpent devore.
20 Hercule, ainsy qu'on dit, feut juste punisseur Des serpents punisseurs et du voleur Antée, De Cacus le Brigand, de l'Hydre surmontée, Du traistre Geryon, du Lion meurtrisseur.

Il vainquit près le Ciel l'esquadron agresseur Et des mutins geans la grande troupe athée ; Du larron Diomede encores feut domptée L'horrible cruauté, et l'esprit ravisseur.

En quelque lieu qu'il feust il surmonta le vice, L'orgueil, la cruauté, l'audace et l'injustice. $\mathrm{Ne}$ sois donc esbahy de voir ces murs vaincus.

Hercule est au milieu, Rome est l'hydre seconde, Les geans, les serpents, un Antée, un Cacus, Geryon, Diomede, et un Lion au Monde.

21 Apollon, s'il est vray ce qu'escrivent les poetes, Espriz (comme l'on dit) de tes sainctes fureurs, Quelque part que tu sois tu portes les malheurs, Les froides pauvretez, ruines et disettes.

Eux, pour estre advouez tes prestres et prophetes,

N'emportent à la fin que travaulx et douleurs, Du plaisir incertain mille sanglots et pleurs, Mille maulx apparents de leurs peines secretes.

Ne t'esmerveille doncq, o ville ruinée

Qui gardes d'Apollon l'image infortunée,

Si tu es maintenant destruite par le temps.

Apollon fut pour Troye et Rhodes sa sujete ; L'une et l'aultre a esté piteusement desfaite, L'une par les Gregeois, l'aultre par les Sultans.

22 O fleuve Egyptien, et toi Tybre mutin, Qui d'un bras recourbé sur la cruche profonde. Versez à flots ondez vostre source feconde, Voyez, voyez les maux de ce peuple Latin ;

Regardez combien peut le temps et le destin ; Rome, qui en grandeur n'a point eu sa seconde, Est faicte la risée et la fable du Monde.

Elle s'est ruinée, et vous estes sans fin.

Vous versez et poussez les ondes montanieres, Qui renaissent tousjours en vos sources premieres,

Pour paroistre au defaut de celle qui se perd.

Rome sans fin poursuit sa course perissante, Mais sa naissance, helas! comme à vous ne luy sert,

Car elle est de ruine une source abondante. 
créé pour Paul III à la fin des années 1530, dont l'architecture est totalement achevée en 1541: les deux Vénus au sonnet 17 désignent le groupe "Venus Felix et Amor" (placée par Jules II en 1503 au Belvédère) ainsi que la Vénus cnidienne ou Vénus au bain (copie de Praxitèle, acquise sous Paul III en 1536); la statue de Cléopâtre aux sonnets 17 et surtout 18 renvoie à la fontaine de Cléopâtre installée par Jules II en 1512 au Belvédère; le Laocoon au sonnet 19 est reconnaissable comme le célèbre groupe statuaire découvert en 1506 et alors placé par Jules II au Belvédère; l'Hercule du sonnet 20 est peut-être l'Hercule Commode placé en 1507 au Belvédère (et en 1536 dans une niche du Cortile), à moins qu'il s'agisse du torse d'Hercule récupéré par Clément VII après le sac de Rome et entré la même année 1527 au Belvédère; l'Apollon du sonnet 21 est l'Apollon du Belvédère, entré dès 1503 dans le Cortile sous Jules II (une des premières statues à y entrer); enfin au sonnet 22, il s'agit des deux statues qui se faisaient face, celle du Nil (entrée au Belvédère avant 1523) et celle du Tibre (entrée au Belvédère avant 1513)³.

Grévin a-t-il vu ces huit statues? il est peu probable qu'un calviniste notoire, à la fin des années 1560, quand bien même ce calviniste faisait partie de la cour du très catholique Emmanuel Philibert de Savoie, ait pu être invité par le pape Pie V à entrer dans le jardin secret, invitation semble-t-il réservée aux hôtes de marque. On peut d'ailleurs remarquer que ces statues font partie de celles qui ont été moulées par Primatice et Vignole (en 1540 et 1545) à la demande de François Ier pour être coulées plus tard à Fontainebleau, toutes à l'exception de la Venus Felix. Est-ce que Grévin les aurait vues en France avant de s'exiler? en avait-il vu des reproductions déjà nombreuses à cette époque? le peu de renseignements sur le séjour probable de Grévin à Rome (seuls ses sonnets nous le disent: le s. 3 surtout: "je passay l'Apennin, je vis Rome deserte") et surtout le peu de détails descriptifs sur les statues (qui ne permet qu'à grand'peine leur identification $)^{4}$ ne permet pas de conclure. En tout cas, il est bien question pour lui de nous conduire, dès le premier sonnet concerné, dans un lieu spécifique: "Voyez des deux Venus la sculpture divine / Au jardin où le pape entre secrettement" (aux v. 3 et 4 du sonnet 17), et c'est cette désignation précise qui fonctionne comme un embrayeur d'ekphrasis, qui nous oblige à lire les huit noms propres ou périphrases qui suivent comme des noms de statues tout autant que comme des noms mythologiques ou historiques. Ce ne sont en revanche pas les éléments descriptifs qui le permettent.

\section{Pratique de l'ekphrasis au XVIe siècle}

S'agit-il alors dans ces six sonnets, d'ekphraseis? Du Bellay, le modèle poétique de Grévin, n'avait pas eu recours à l'ekphrasis dans Les Antiquités; en revanche, il s'y est livré plusieurs fois dans les Poemata et dans Le Songe qui sont aussi des recueils romains: par exemple, le sonnet 9 du Songe dans son entier et l'élégie III (vv. 1-34), de manière ponctuelle, procèdent par ekphraseis du Tibre (c'est-à-dire de la statue du Tibre, telle qu'on peut la voir au Belvédère); l'élégie II, quant à elle, procède par

(3) Tous les renseignements historiques ainsi que les représentations des statues se trouvent dans le livre précieux de P. P. Bober et R. O. Rubinstein, Renaissance Artists and Antique Scuplture, Harvey Miller Publishers, 1991 (e. o. 1986). L'article de S. Deswarte-Rosa Sur Francisco de Hollanda et le Cortile di Belvedere m'a aussi été utile pour comprendre la progression de la collection et son état vers 1538-1540, article publié in Il Cortile delle Statue, Verlag Philip Von Zabern, Mainz, 1998, pp. 389-410.

(4) J'avoue ne pas avoir vu qu'il s'agissait de statues quand j'ai édité le texte, ce qui révèle le caractère très discret de l'ekphrasis ici. Je remercie Gilles Polizzi d'avoir attiré mon attention sur ces statues. 
une juxtaposition d'ekphraseis de statues'. C'est peut-être là que Grévin a trouvé son modèle pour la pratique de l'ekphrasis, pratique par ailleurs très répandue au XVIe siècle. Il a pu lire aussi l'ekphrasis néo-latine très célèbre de Jacques Sadolet sur le Laocoon (De Laocoontis Statua $)$ ou celles des Stanze d'Eurialo d'Ascoli, ornées de bois gravés du Laocoon, de la Vénus au bain et de l'Apollon ${ }^{7}$, ou, plus proches dans le temps, peut-être a-t-il lu le livre d'Ulysse Aldrovandi publié à Rome en 1556, Tutte le statue antiche che in Roma si veggono: appendice aux Antichita de Lucio Mauro, celui de Cavalieri en 1562, Antiquae statuae Urbis Romae, celui d'Achille Statius en 1569 (peut-être exactement contemporain de son séjour romain): Inlustrium virorum ut exstant in urbe expressi vultus) ? il avait pléthore d'ouvrages à sa disposition à cette date pour voir représentées ces statues, s'il ne les a pas vues lui-même au Vatican.

Grévin est médedin et il a pu aussi avoir accès à l'ekphrasis via l'œuvre de Galien qu'il a lu pendant ses études de médecine : Galien a souvent recours à la comparaison entre la statuaire et le corps humain ${ }^{8}$, ce qui lui permet de nombreuses ekphraseis, et d'autre part, Grévin a commenté, d'abord en latin en 1564, puis en français en 1569, une édition des Portraicts anatomiques de toutes les parties du corps bumain, ensemble l'abbregé d'André Vesal, qui était constitué de 39 planches anatomiques en taille douce; donc le commentaire du corps représenté ne lui était pas une pratique étrangère.

\section{Définitions de l'ekphrasis}

Si la pratique de l'ekphrasis est répandue à la Renaissance, c'est que l'on redécouvre les grandes ekphraseis antiques? et surtout les auteurs de la seconde sophisitique, grands praticiens de l'ekphrasis, dont les œuvres de Lucien ${ }^{10}$ et les Tableaux de platte peinture de Philostrate que donnera Blaise de Vigenère en 1578 sont deux des exemples les plus manifestes ${ }^{11}$.

(5) La statue du Tibre qui est ici le support des ekphraseis de Du Bellay est aussi celle que décrit Vigenère en donnant les Images ou tableaux de platte-peinture de Philostrate (I, 5) [voir l'éd. de F. Graziani, Paris, Champion, 1995, p. 91], Vigenère qui précise que la statue se trouve au Belvédère. Les ekphraseis du Tibre dans les deux élégies latines de Du Bellay ont été étudiées par P. GALAND-HALLYN dans Les Yeux de l'éloquence, poétiques humanistes de l'évidence, Orléans, Paradigme, 1995, pp. 163 171: elle parle d'ailleurs tantôt d'hypotypose (p. 163), tantôt d'ekphrasis (p. 164 et 165). Quant aux autres ekphraseis de statues dans la "Romae Descriptio", vv. 80-104, elles ont pu servir de modèles littéraires à Grévin (sont évoqués successivement Apollon, la Vénus Cnidienne, l'enfant à l'épine, la Louve, Romulus et Rémus, Hercule, le Tibre, Cléopâtre, Mars et Vénus, un satyre, Adonis,...). G. DEMERSON dans sa traduction annotée de l'élégie précise qu'il s'agit d'une "série d'ekphraseis rapides", in CEuvres poétiques, Poemata, tome VII, STFM, Paris, Nizet, 1984 , note p. 228 , ce qui n'est pas sans rapport avec la pratique de Grévin.

(6) Ce poème, édité pour la première fois en 1532, fut écrit dès la découverte du Laocoon en 1506; on en trouve le texte latin et sa traduction italienne dans l'ouvrage de S. SeTTIS, Laocoonte, Donzelli editore, 1999 , pp. 118-121 et une traduction française dans Musae reduces, Anthologie de la poésie latine dans l'Europe de la Renaissance, éd. P. Laurens et Cl. Balavoine, Leiden, Brill, 1975, T. I, p. 211 et $s q$.

(7) Voir l'article de S. Deswarte-Rosa, cité plus haut, pp. 394-395.

(8) Voir à ce sujet l'article de V. Boudon, Galien et les arts figurés: un médecin amateur d'art, in La Littérature et les arts de l'Antiquité à nos jours, $\mathrm{Pa}$ ris, Les Belles Lettres, 2001, pp. 209-217.

(9) Du bouclier d'Achille (chant XVIII de l'Iliade, vv. 665-854) au bouclier d'Enée (chant VIII de l'Enéide, vv. 626-731), en passant par la toile de Pallas et celle d'Arachné dans les Métamorphoses d'Ovide (VI, 70-130), le tableau de la prise de Troie commenté en vers par Eumolpe dans le Satiricon de Pétrone (89), la scène pastorale décrite sur une bague dans les Ethiopiques d'Héliodore (traduit par Amyot en 1559), celle du tableau d'Europe dans les Aventures de Leucippé et Clitophon d'Achille Tatius...

(10) Voir à ce sujet l'article de J. Bompaire, Regards d'écrivains grecs sur la sculpture au temps de la seconde sophistique, in La Littérature et les arts de l'Antiquité à nos jours, Paris, Les Belles Lettres, 2001, surtout pp. 203-207.

(11) Dès 1503, Alde Manuce donnait à Venise dans les Luciani Opera les éditions réunies des Icones Philostrati, Icones junioris philostrati et des descriptiones Callistrati: trois séries d'ekphraseis 
Mais tous ces poètes français du XVIe siècle praticiens de l'ekphrasis, qu'il s'agisse de Ronsard, de Du Bellay, de Belleau, de Pontus de Tyard ou ici de Grévin, n’ont pas eu de théorie à laquelle s'adosser. Les arts poétiques et les rhétoriques de l'époque restent muets quant au mot ekphrasis, de même que les arts poétiques et les traités latins sur l'éloquence, qu'il s'agisse de l'Art poétique d'Horace, des traités de Cicéron aussi bien que l'Institution oratoire de Quintilien (si Quintilien parle d'hypotypose et d'enargeia, il n'emploie pas le mot "ekphrasis" ${ }^{12}$ ). La notion est rare, le mot quasi absent, la pratique fréquente.

On sait que l'ekphrasis a été théorisée par les orateurs de la seconde sophistique comme Aelius Théon dans ses Progymnasta:

La description (ekphrasis) est un discours qui présente en détail et met sous les yeux de façon évidente ce qu'il donne à voir [...] les vertus de la description sont avant tout les suivantes: la clarté et l'évidence (enargeia) qui fait presque voir ce qu'on présente, puis de ne pas s'étendre tout au long sur les détails inutiles ${ }^{13}$.

\section{On retrouve exactement la même définition chez Hermogène de Tarse:}

L'ekphrasis est un discours descriptif détaillé, vivant et mettant sous les yeux ce qu'il montre. On réalise des ekphraseis de personnes, d'actions, de circonstances occasionnelles, de lieux, de saisons, et de beaucoup d'autres sujets [...] les vertus de l'ekphrasis sont principalement la clarté et l'illusion de vie (enargeia) ${ }^{14}$.

La définition de l'ekphrasis à l'origine est large (c'est celle de la descriptio, ne se distinguant pas vraiment de l'hypotypose) et elle ne correspond pas à la définition étroite aujourd'hui retenue de "description verbale d'une œuvre non verbale" ${ }^{15}$, définition que choisit François Lecerclepour la Renaissance : "l'ekphrasis est une description, en prose ou en vers, d'une œuvre plastique - statue ou tableau, voire objet décoratif - réelle ou imaginaire" ${ }^{16}$. C'est bien celle que nous retiendrons.

Je n'ai retrouvé que de rares occurrences de la notion sous la plume des humanistes au XVIe siècle-elles vont presque dans le même sens que les rhéteurs antiques - dans les Antiquarum lectionum libros de Caelius Rhodiginus ${ }^{17}$, dans le Thesaurus linguae latinae de Robert Estienne (qui reprend C. Rhodiginus), dans les Images ou Tableaux de platte-peinture de Philostrate traduits et commentés par Vigenère et dans le dictionnaire de Cotgrave ${ }^{18}$ :

datant de la seconde sophistique. Trois textes qu'on retrouve dans l'édition augmentée de Vigenère en 1614.

(12) Voir Institution oratoire, IX, 2, 40-44: interviennent à proximité les unes des autres, hypotypose (evidentia), enargeia et topographie; la notion d'ekphrasis qui aurait eu sa place ici n'apparaît pas.

(13) THÉON, Progymnasta, 118-120, “peri ekphraseos", éd. M. Patillon, Paris, Les Belles Lettres, 1997, pp. 66-69.

(14) Hermogène de Tarse cité par P. GalandHallyn, Les Yeux de l'éloquence, op. cit., p. 100.

(15) Celle que retient G. MolinIÉ dans son Dictionnaire de rbétorique, Le Livre de poche, 1992, un des rares dictionnaires actuels à donner une entrée "ecpbrasis"; ni le TLF, ni le Grand Robert, ni un dictionnaire des figures de style comme le Gradus ne mentionnent l'ekphrasis, ce qui prouve qu'il y a un problème d'acclimatation en français de la notion; le mot est défini dans l'Oxford Classical Dictionary.

(16) F. LECERCLE au chapitre VIII “ Arts et littérature" du Précis de littérature française, PUF, 1991, p. 360. Il fait de même dans La Chimère de Zeuxis, Gunter Narr Verlag Tübingen, 1987, pp. 20-22.

(17) J'ai consulté l'édition originale, celle des héritiers d'Alde Manuce à Venise en 1516, pas encore complète des trente livres, mais comportant bien au chapitre 39 du livre 7 la définition de l'ekphrasis que cite R. Estienne (quoiqu'il renvoie au livre 13, chapitre 19).

(18) Dans son Dictionarie of the French and English Tongues, 1611, on a une entrée "ecphrase" (le même mot que celui employé par Vigenère) avec cette définition laconique: "a plaine declaration, or exposition". 
L'objet de Caelius Rhodiginus est de distinguer "ekphrasis" et "diegema" (=récit, narration), comme le signale un fragment du titre du chapitre qui concerne cette notion: "ekphrasis quo a diegemate differt" et c'est bien l'idée que reprend Estienne dans son Thesaurus en le citant. On voit que l'ekphrasis est déjà distinguée de la narration (diegema) par rapport à laquelle elle est perçue comme un élément hétérogène.

Selon Robert Estienne:

Ecphrasis: est Mera puraque ennaratio, [apo tou ekphrazein], quod est exprimere, clare et evidenter explicare. Hesychius [logon enarge], h.e. sermonem evidentem interpretatur.

Ecphrasis autem, ut Caelio placet, a narratione differt: quod haec nudam habeat rerum expositionem; illa tantum non etiam oculis audientium subjiciat. Praetaera Narratio universim inquirit: sicuti, in Athenienses a Lacedemoniis esse bellatum. Ecphrasis autem, per partes, utiquo genere belli, quibusque machinarum tormentis. Haec ille Ant. Lect. Lib. 13 cap. $19^{19}$.

Quant à Blaise de Vigenère, en 1578, dans son "Epître à Barnabé Brisson", épître liminaire de sa traduction des Images ou tableaux de platte peinture, il francise le mot et en donne un définition très intéressante:

Tout y est plein de Prosopoeïes, Hypotyposes, et Ecphrases: nous ne savons comme bonnement appeler ces fictions de personnes: représentations au naturel: et descriptions tresnaïves qui nous introduisent les choses plus distinctement en l'appréhension; nous les approchent trop mieux du sentiment; et les impriment plus vivement en la cognoissance que tous les chefs d'œuvre de peinture et imagerie qui soient oncques partis des plus anciens souverains maitres ${ }^{20}$.

On remarque que les trois premiers termes se distribuent dans les trois définitions qui suivent. Et l'intérêt vient du distinguo, difficilement conceptualisable mais posé, entre hypotypose et ekphrasis. L'ekphrasis est spécifique: elle n'est pas qu'evidentia ou enargeia: elle n'est pas qu'une mise sous les yeux d'une illusion de vie. Mais qu'est-elle?

Pour ces hommes de la Renaissance, l'ekphrasis ne se définit pas par son sujet, comme pour nous aujourd'hui, mais par sa forme et sa fonction: c'est une description qui se dissocie de la narration et provoque un effet de "naïveté", c'est-à-dire de vie, mais pas comme l'hypotypose le fait. Aucun n'assigne à l'ekphrasis la description d'une œuvre d'art, même si la chose est finalement implicite pour Vigenère.

On sait avec les trois définitions données plus haut (entre 1516 et 1578) qu'ekphrasis n'est ni hypotypose ni narration et que le discours sur l'ekphrasis s'entretisse inévitablement avec celui de la supériorité de la poésie sur les arts plastiques, comme le pose Vigenère, ce qui est un des grands débats du XVIe siècle, très vif en France surtout au sein de la Pléiade et explique le peu de représentativité de l'ekphrasis avant $1530^{21}$ et le peu de théorisation de cette pratique quand elle se développpe après $1530^{22}$.

(19) Thesaurus linguae latinae, reproduction anastatique de l'éd. de Bâle, 1740, tome II, "Culture et civilisation”, Bruxelles, 1964, (e.o. 1536). En revanche, on ne trouve pas d'entrée "ecphrasis" dans son Dictionarum latinogallicum (éd. consultée: Paris, 1543). Rien dans Nicot non plus.

(20) B. DE Vigenère, Images ou Tableaux de platte peinture de Philostrate, op. cit., t. I, p. 8.

(21) Voir l'analyse que fait P. GALAND-HaLlyn des raisons de la sous-représentation de l'ekphrasis en France avant 1530 dansLes Descriptions d'art dans la poésie néo-latine, du Quattrocento au début de la Renaissance française, in La Littérature et les arts de l'Antiquité à nos jours, Paris, Les Belles Lettres, 2001, pp. 539-553.

(22) Les sonnets 5 et 25 des Antiquités par exemple théorisent la supériorité de la poésie sur l'architecture. 


\section{Résistance à l'ekphrasis?}

Les humanistes parlent - très rarement - d'ekphrasis et toujours sans recourir à la notion, sans la nommer: c'est le cas de Barthélemy Aneau dans la préface de l'Imagination poétique en 1552 (entreprise fondée sur l'ekphrasis) et c'est le cas de Ronsard dans la seconde préface de La Franciade. Aneau raconte qu'il découvre chez Macé Bonhomme, imprimeur lyonnais de ses amis, "quelques petites figures pourtraictes" (des bois gravés) qui ne servent à rien à l'imprimeur qui n'a pas "d'inscriptions propres à icelles”; “alors je estimant que sans cause n'avoient esté faictes, luy promis que de muetes, et mortes, je les rendray parlantes, et vives: leur inspirant âme par vive Poësie [...] appropriant non les images aux parolles (comme il falloit) mais les paroles aux images (comme j'estois contrainct) les plus convenables qu'il me a esté possible. Affin que les images ensevelies, et muetes, je ramenasse en lumiere et en vie"23. On retrouve ici la même supériorité des paroles sur les images que chez Vigenère, avec une nuance supplémentaire: c'est aux images de servir la parole et non l'inverse: le recours à l'ekphrasis dans le cadre de l'Imagination poétique relève d'un cas de force majeure, B. Aneau pratique par défaut l'ekphrasis. Il en va pour Aneau comme pour Ronsard dans les Odes: la supériorité du poétique sur le non-verbal, fruit des arts muets et, de ce fait, inférieurs est un préalable :

C'est lui ["mon mettre"] qui mieus te fera vivre

Qu'un portrait de marbre attaché;

Ou qu'une medaille de cuivre

Mise à ton los dans un marché

(“A Charles de Pisseleu”, Odes, II, 18, O.C., éd Laumonier, p. 227)

Je n'ai les mains apprises

Au métier muet de ceus,

Qui font une image assise

Sus des piliers paresseus

Ma peinture n'est pas mue

Mais vive $[\ldots]$

(A René d'Urvoi, Odes, IV, 17, O.C., p. 148)

Malgré (ou à cause de) cette dépréciation des arts plastiques, le même Ronsard recommande sans la nommer l'ekphrasis dans la préface de La Franciade: le poète hérö̈que doit illustrer "la matière qu'il s'est proposé d'escrire, tantôt par personnage parlans les uns aux autres, tantost par songes, prophéties et peintures insérées contre le dos d'une muraille et des harnois, et principalement des boucliers ${ }^{24}$, ou par les dernières paroles des hommes qui meurent ou [etc] "25. Dans cette énumération des moyens exceptionnels auxquels peut recourir le récit hérö̈que, intervient donc l'ekphrasis mais de manière empirique, (avec l'évocation des trois supports), comme si Ronsard n'avait pas le concept à sa disposition (ce qui est fort improbable). Si elle est attendue dans le poème héroïque (et ses avatars: le roman antique et le romanzo italien), l'intervention de l'ekphrasis en poésie lyrique (son lieu majeur), pratiquée par exemple par Ronsard dans les Odes, par Du Bellay dans les Poemata, par Belleau dans La Bergerie ${ }^{26}$ et ici, par Grévin, n'est en revanche pas théorisée dans les arts poétiques français.

(23) Préface de l'Imagination poétique, Lyon, Macé Bonhomme, 1552, p. 6 et 8.

(24) C'est moi qui souligne.

(25) O.C., op. cit., La Franciade, Tome XVI, p. 336.

(26) Voir l'article de R. CAMPO:Du Miroir à la mémoire: sur les jeux ecphrastiques dans La Berge- rie de Remy Belleau, Nouvelle Revue du Seizième siècle, 2002, n²0/2, pp. 5-23 et plus généralement pour tous les auteurs de la Pléiade, l'ouvrage de F. Joukovsky, Le Bel Objet. Les Paradis artificiels de la Pléiade, Paris, Champion, 1991. 
Il est clair que c'est dans ce contexte de défiance quant aux arts non-verbaux, de supériorité du verbal sur le visuel que se situe Grévin, qui d'être calviniste, est encore un peu plus prévenu que des catholiques contre le statut des images.

Les six sonnets montrent leur résistance propre à l'ekphrasis: ils l'induisent tout en s'y refusant. Des indices prouvant qu'il s'agit des sculptures du Belvédère sont disposés dans les sonnets, mais tout est fait en même temps pour contrarier leur représentation. Les indices indiscutables sont dans le sonnet 17 (vv. 3-4) et dans le sonnet 22 (vv. 1-3), c'est-à-dire dans les deux sonnets qui encadrent la série ; en revanche, dans les sonnets intermédiaires, rien ne permet l'identification des statues (sinon l'expression “D'Apollon l'image infortunée"); l'ekphrasis est minimale, fonctionnant sur le nom propre et de très rares éléments descriptifs (rien sur les Vénus sinon leur identification comme statues, rien sur Cléopâtre, rien sur Hercule, rien sur Apollon): seuls le Laocoon et les deux fleuves, le Nil et le Tibre, reçoivent quelques qualifications descriptives. Mais là encore il faut distinguer entre le traitement du Laocoon et celui des deux fleuves: ce dernier est indiscutablement ekphrastique puiqu'on voit au vers 2 et 3 le geste effectué par les deux fleuves tels qu'ils ont été scupltés (on retrouve la même posture chez Du Bellay: voir, outre les Poemata, le Songe au sonnet 9 et son illustration (postérieure) qui est elle-même une représentation, dans un décor naturel, de la statue du Tibre ${ }^{27}$; on retrouve aussi la même chose chez Vigenère). On peut noter chez Grévin, dans l'expression "d'un bras recourbé sur la cruche profonde” au sonnet 22, v. 2, l'importance de l'article défini "la" à valeur de notoriété, qui renvoie de toute évidence à un objet connu de tous.

Pour le Laocoon, le trouble vient de ce que deux scènes (d'ailleurs plus narratives que descriptives) se succèdent: au premier quatrain, le geste de Laocoon frappant le cheval de Troie (Enéide, II, 40-49) et dans le deuxième quatrain, la mort de Laocoon étouffé par les serpents (Enéide, II, 201-207): seule la deuxième scène peut être ekphrastique mais le modèle virgilien textuel tend à l'emporter sur la référence sculpturale. A lieu ici une double imitation simultanée, du poème et de la sculpture, manière d'accentuer la pression du verbal sur le non-verbal, car à l'origine et à l'aboutissement, il y a du texte, et entre les deux la possibilité plastique, à peine suggérée par les sonnets environnants.

Comme l'a démontré P. Galand-Hallyn à propos de l'ekphrasis du Tibre dans l'élégie III de Du Bellay, le modèle originel d'une ekphrasis peut, à la Renaissance, être tout autant plastique que textue ${ }^{28}$, et dans le cas précis de Du Bellay empruntant d'ailleurs plus à Ovide (Mét. I, 568-582) qu'à Virgile (Enéide, VIII, 31-34 ou même Enéide VII, 792).

L'ekphrasis est non seulement le lieu de confrontation de deux systèmes sémiotiques différents (verbal et non-verbal), elle est aussi le lieu de confrontation de deux cultures, sujet essentiel du tableau de la Rome contemporaine, doublé d'un tableau de la Rome antique, diptyque que forment aussi bien les poèmes romains de Du Bellay que les vingt-quatre sonnets de Grévin.

\section{Fonction de cette ekphrasis suggérée}

L'ekphrasis est en général associée à une reconnaissance de la valeur esthétique de l'objet décrit et, de façon corollaire, au tour de force verbal que représente la ten-

(27) Voir l'illustration qu'en donne M. SCREECH dans son édition des Regrets et autres ouvres poétiques, Genève, Droz, 1979 (encart après la page 316).

(28) Les Yeux de l'éloquence, op. cit., p. 169: “ob- jet de l'ekphrasis de Du Bellay, le Tibre est donc présenté tantôt comme une allégorie textuelle à la mode antique, tantôt comme la figuration plastique de cette allégorie, la statue d'un vieillard accoudé à son urne". 
tative de description de cette excellence: on se souvient de l'annonce de Virgile quant au bouclier d'Enée(Enéide, VIII, 625) "Et clipei non ennerabile textum" " "bouclier à la texture innénarrable”). C'est dire que l'ekphrasis relève de la rhétorique épidictique et qu'elle est en général associée à l'éloge ${ }^{29}$.

Chez Grévin, rien ne relève de l'éloge: les plus belles sculptures qui font s'extasier les humanistes de l'Europe entière n'engendrent chez lui qu'une rhétorique du blâme qui va appuyer une satire de la Rome papale. De façon explicite, Laocoon est un "signe ruineux"(s. 19, v. 11), ce qui laisse entendre que Grévin ne voit pas la sculpture (signe évidemment magnifique) mais ce qu'elle représente (signe de la ruine de Troie); la statue d'Apollon est, elle aussi de façon tout aussi paradoxale, une “image infortunée" (s. 21, v. 10); L'emploi du mot "signe" d'ailleurs révèle immédiatement la portée allégorique des ekphraseis ici; comme l'annonce le premier sonnet de la série, les statues sont une réponse pour ceux qui cherchent les causes de la ruine de Rome. Ni signe d'excellence artistique, ni signe d'excellence verbale, l'ekphrasis a pour Grévin un sens allégorique, elle sert à illustrer un argument:elle est ici doublement allégorique, valant pour la Rome antique et pour la Rome papale. Ainsi Laocoon est un signe de ruine pour Troie et un signe de ruine pour Rome, comme le dit le dernier tercet du sonnet 19 qui fonctionne sur une ambiguïté: le "vieil Laocoon que le serpent dévore” pouvant aussi bien être la statue du Belvédère qu'une métaphore pour désigner le Pape, ce qu'induit le vers final du sonnet précédent, "lemoine" désignant par métonymie le pape Pie V. Une continuité s'établit dans cinq des six sonnets entre l'univers antique et la situation actuelle de Rome et ce sont les statues qui en sont les signes évidents (pas seulement allégoriques) puisque, physiquement, elles appartiennent à l'Antiquité et participent du présent de Rome où elles trônent. Elles sont la possibilité de revivre les événements du passé: si Hercule est à Rome (sous la forme d'une statue), c'est que les monstres à abattre sont à Rome (s. 20: noter le passage du passé simple dans les trois premières strophes au présent de la dernière). Apollon favorable à Troie et Rhodes n'empêcha pas leur prise par l'ennemi: c'est donc que la présence d'Apollon dans les murs de Rome annonce la même issue; pour les deux Vénus du sonnet 17, elles sont d'abord le signe d'une lubricité papale puis se transforment en la série "Cléopâtre, Venus, Flore, Faustine", qui allégorise le féminin comme principe de tentation, principe finalement illustré par le couple "une Vénus et une Heleine", pour finir, grâce à l'antonomase du vers final, par représenter toutes les femmes tentatrices, (c'est-à-dire "une infinité”): les deux statues de Vénus ne faisaient que préparer l'irruption vivante de ce qu'elles désignent allégoriquement: la femme comme principe de perdition. Il ne s'agit pas de soigner l'ekphrasis au point que l'on croie à une transparence du signe et que Vénus devienne vivante sous nos yeux (finalité idéale de l'ekphrasis), il s'agit seulement de préparer l'apparition du vivant déjà codé dans le signe-allégorie: "dans Rome on voit/ Presqu'une infinité de Venus et d'Heleines"; nous sommes devant un discours moral qui ne se soucie pas de la valeur esthétique des signes (pas plus des signes plastiques que des signes verbaux d'ailleurs: c'est la grande différence entre Grévin et Du Bellay). Voir, c'est comprendre, et ce, dès le premier sonnet qui établit un lien entre une recherche intellectuellle (v. 2) et une vision qui, de sensible, devient immédiatement intellective: Pour Grévin, il faut surtout ne pas s'arrêter sur les apparences, ne pas s'enivrer d'apparences comme certaines ekphraseis appellent à le faire avec les illusions qu'elles engendrent dues au vertige des signes se substituant au réel, devenant le réel ${ }^{30}$.

(29) Voir l'ouvrage de M. BAXANDALL, Les Humanistes à la découverte de la composition en peinture, 1340-1450, Paris, Seuil, 1989, pp. $111-114$ et p. 117-124.
(30) Voir dans les Images de Philostrate, op. cit., les illusions créées par les représentations en I, 22, I, 25, I, 27... 
Preuve qu'il ne s'agit pas de s'enivrer d'apparences, mis à part le sonnet 17 et le sonnet 20 (v. 11), quand le verbe "voir" est utilisé, il en appelle au regard des statues: cela repose sur un déplacement énonciatif: il ne s'agit plus de s'adresser au lecteur-spectateur (comme dans le sonnet 17) mais aux statues elles-mêmes: "tu la vois" et "te faict voir" sont les verbes associés à Cléopâtre au sonnet 18; "voyez, voyez" et "regardez" sont les impératifs adressés aux deux fleuves du sonnet 22: c'est à ce point que l'ekphrasis devient paradoxale: elle rend voyant les objets que l'on est censés voir, prouvant que le spectacle est ailleurs. Si l'art est vivant (indice d'ekphrasis normalement) c'est non grâce aux prodiges de la description mais grâce à l'adresse lyrique (un pur procédé énonciatif) qui fait des statues des êtres vivants.

Que cette ekphrasis relève du blâme plus que de l'éloge et qu'elle soit allégorique (la valeur herméneutique de l'ekphrasis est fréquente) ne sont pas des éléments surprenants, mais que l'œil ne soit pas appelé à voir, que la description ne se développe pas, que l'ekphrasis reste une pure allusion sont en revanche des éléments perturbants.

Qu'elle soit, en dernier lieu, associée à un écriture burlesque est encore plus étrange. Le burlesque (association d'un sujet noble et d'un style bas) apparaît avec la mention d'Enée, fils d'une concubine au sonnet 17 : cette concubine est précisément Vénus dont on vient d'évoquer "la sculpture divine" : la chute du divin vers le trivial est nette, préparant la métamorphose de la déesse en putain (vers final); le burlesque continue avec "le vieil Laocoon" du sonnet 20 si l'on y voit une caricature du Pape livré aux serpents de la tentation, et avec Apollon présenté en dieu de malheur, assurant la ruine de ceux qu'il protège, qu'il s'agisse des poètes ou des cités.

Le recours à la satire et au burlesque pour dégrader la Rome catholique du XVIe siècle n'est pas chose nouvelle: Du Bellay l'a fait dans Les Regrets mais utiliser l'ekphrasis afin de construire cette satire burlesque de la Rome des années 1560 est plus surprenant: la magnificence de la statuaire antique sert à révéler une Rome luxurieuse, rongée par le vice, piteusement ruinée et attachée à sa ruine. Si les preuves les plus irréfutables de l'art humain que sont les statues du Belvédère n'ont que valeur de désignation de la méchanceté et de la vanité humaines, c'est qu'indirectement une réflexion sur l'art est induite par le texte.

C'est le dernier sonnet de la série qui en est le lieu: plus de burlesque ici mais une réflexion philosophique grave sur la nature, opposée aux réalités humaines. Dans ce sonnet, les statues du Nil et du Tibre exaltent un principe contraire à ce qu'elles sont: l'art dit le triomphe de la nature sur l'art. La thèse est néoplatonicienne: la critique des simulacres est toujours sous-jacente chez Grévin. Les deux statues, pourtant clairement reconnaissables de ce sonnet 22, ne sont pas des statues mais désignent les fleuves dans leur réalité physique: l'art est oublié, non pas dans le triomphe paradoxal du signe qui viserait à sa transparence, mais dans sa réelle nullité qui le fait s'évanouir devant son référent et plus encore devant l'interprétation allégorique de ce référent : la nature est dans un perpétuel recommencement (une renaissance qui annule la perte):

Vous versez et poussez les ondes montanières,

Qui renaissent tousjours en vos sources premières,

Pour paraistre au defaut de celle qui se perd

Quand Rome ne fait que confirmer sa perte :

(31) Peut-on y voir un appel iconoclaste à briser les statues? sans doute pas, mais l'implicite de l'argument: (sans la présence de ces statues en son sein, Rome irait moins à sa perte) est tout de même lourd de sens. 
Rome sans fin poursuit sa course périssante

Mais sa naissance, helas! comme à vous ne lui sert

Car elle est de ruine une source abondante.

L'art ne témoigne donc d'aucune valeur humaine: il peut, dans une sorte d'ironie tragique, désigner la puissance de la nature et l'inanité humaine, telle que l'indique la transformation métaphorique de "source", à visée satirique dans la chute du sonnet. La "copia” (abondance verbale), impossible dans la description des statues, existe dans cette corne d'abondance inversée qu'est Rome, productrice infinie de ruine.

Grévin ne se livre dans les Vingt-quatre sonnets romains qu'à une esquisse d'ekphrasis; si ekphrasis il y a, elle n'existe que par la série: elle est suggérée par la galerie de statues que constituent les six sonnets enchaînés et par l'énonciation qui prête vie et intention à ces statues; il pourrait donc - c'est là l'originalité - y avoir ekphrasis sans description mais seulement par désignation des œuvres et choix énonciatif.

Dans une topographie romaine qui n'est qu'énumération de débris, la série du Belvédère semble se détacher en montrant des objets intègres (ou quasi intègres), vivants et admirables, ce que laisse croire un instant la mention de la "sculpture divine" au début de la série. L'ambiguïté de l'adjectif “divine”, qui peut aussi bien être un constat objectif qu'un éloge, est vite levée: il ne s'agit que de la sculpture d'une déesse, déesse aussitôt dépréciée. La sculpture n'est pas divine par essence, la poésie ne cherche pas à rivaliser avec elle, et la beauté se fait le signe de la laideur morale. Aucune des caractéristiques attendues de l'ekphrasis n'apparaît. Seule une lecture allégorique à finalité satirique se développe: la Rome papale est ici accusée de garder en son sein ("Au jardin où le pape entre secrettement") les signes de sa vilenie et de sa ruine ${ }^{31}$. Le triomphe de l'art signe le désastre de la Rome catholique. Et c'est parce que cet art est signe de désastre, qu'il ne peut y avoir complaisance à le décrire, ce qui explique le surgissement de cette étrange ekphrasis laconique. 


\section{Bibliographie}

M. BaXANDAll , Les Humanistes à la découverte de la composition en peinture, 1340-1450, Paris, Seuil, 1989.

P. P. Bober et R. O. Rubinstein, Renaissance Artists and Antique Scuplture, Harvey Miller Publishers, 1991 (e. o. 1986)

R. CAMPO, Du miroir à la mémoire: sur les jeux ecphrastiques dans La Bergerie de Remy Belleau, «Nouvelle Revue du Seizième siècle», 2002, n²0/2, pp. 5-23

K. CsürÖs, La Fonction de l'ekphrasis dans les longs poèmes in « Nouvelle Revue du XVIe siècle » 51/1, 1997, pp. 169-183.

S. Deswarte-Rosa ,Francisco de Hollanda et le Cortile di Belvedere in « Il Cortile delle Statue », Verlag Philip Von Zabern, Mainz, 1998, pp. 389-410.

PH. FORD , La Fonction de l'ekphrasis chez Ronsard in « Ronsard en son quatrième centenaire », Genève, Droz, 1989, THR n CCXXXII, pp. 81-89

P. Galand-Hallyn, Le Reflet des fleurs. Description et métalangage poétique d'Homère à la Renaissance, Genève, Droz, 1994, THR n CCLXXXIII.

P. Galand-Hallyn, Les Yeux de l'éloquence. Poétiques bumanistes de l'évidence, Orléans, Paradigme, 1995

F. Joukovsky, Le Bel Objet. Les Paradis artificiels de la Pléiade, Paris, Champion, 1991

M. KRIEGER, Ekphrasis :the illusion of natural sign, Baltimore, Johns Hopkins Université Press, 1992

F. LECERCLE , La Chimère de Zeuxis, Gunter Narr Verlag, Tübingen, 1987.

G. Molinié, Dictionnaire de rhétorique, Le Livre de poche, 1992

La Littérature et les arts de l'Antiquité à nos jours, Paris, Les Belles Lettres, 2001 (nombreux articles sur l'ekphrasis) 